\title{
Left Hemisphere Diffusivity of the Arcuate Fasciculus: Influences of Autism Spectrum Disorder and Language Impairment
}

\author{
T.P.L. Roberts, K. Heiken, D. Zarnow, J. Dell, L. Nagae, L. Blaskey, C. Solot, S.E. Levy, J.I. Berman, and J.C. Edgar
}

\begin{abstract}
BACKGROUND AND PURPOSE: There has been much discussion whether brain abnormalities associated with specific language impairment and autism with language impairment are shared or are disorder specific. Although white matter tract abnormalities are observed in both specific language impairment and autism spectrum disorders, the similarities and differences in the white matter abnormalities in these 2 disorders have not been fully determined.
\end{abstract}

MATERIALS AND METHODS: Diffusion tensor imaging diffusion parameters of the arcuate fasciculus were measured in 14 children with specific language impairment as well as in 16 children with autism spectrum disorder with language impairment, 18 with autism spectrum disorder without language impairment, and 25 age-matched typically developing control participants.

RESULTS: Language impairment and autism spectrum disorder both had (elevating) main effects on mean diffusivity of the left arcuate fasciculus, initially suggesting a shared white matter substrate abnormality. Analysis of axial and radial diffusivity components, however, indicated that autism spectrum disorder and language impairment differentially affect white matter microstructural properties, with a main effect of autism spectrum disorder on axial diffusivity and a main effect of language impairment on radial diffusivity.

CONCLUSIONS: Although white matter abnormalities appear similar in language impairment and autism spectrum disorder when examining broad white matter measures, a more detailed analysis indicates different mechanisms for the white matter microstructural anomalies associated with language impairment and autism spectrum disorder.

ABBREVIATIONS: $A D=$ axial diffusivity $\left(\lambda_{1}\right)$ [where $\lambda_{1}, \lambda_{2}$, and $\lambda_{3}$ are the eigenvalues of the diffusion tensor]; ASD $+\mathrm{LI}=$ autism spectrum disorder with significant clinical language impairment; ASD-LI = autism spectrum disorder without language impairment; CELF-4 = Clinical Evaluation of Language-Fourth Edition; FA = fractional anisotropy; $\mathrm{MD}=$ mean diffusivity $\left(\lambda_{1}+\lambda_{2}+\lambda_{3}\right) / 3$ [where $\lambda_{1}, \lambda_{2}$, and $\lambda_{3}$ are the eigenvalues of the diffusion tensor]; RD $=$ radial diffusivity $\left(\lambda_{2}+\lambda_{3}\right) / 2$ [where $\lambda_{1}, \lambda_{2}$, and $\lambda_{3}$ are the eigenvalues of the diffusion tensor]; SLI = specific language impairment

$\mathbf{S}$ imilarities and differences in the biologic "substrates" of language impairment (LI) in patients with specific language impairment (SLI) and in language-impaired patients with autism spectrum disorder (ASD) have been the subject of debate for nearly 30 years. ${ }^{1}$ Some argue that the LI observed in both disorders is similar in nature. ${ }^{2,3}$ Supporting this view, adolescents with a history of SLI have a 10-fold increased risk for ASD. ${ }^{4}$ These 2 groups also share anatomic and electrophysiologic abnormalities. For example, patients with SLI and those with ASD with LI (ASD+LI) both have reversed asymmetry in frontal language-related cortex ${ }^{5}$ as well as delayed auditory-

Received April 23, 2013; accepted after revision June 24.

From the Lurie Family Foundations MEG Imaging Center, Department of Radiology, Children's Hospital of Philadelphia, Philadelphia, Pennsylvania.

This work was supported by NIH-R01DC008871 and NIH-P30HD026979 grants.

Please address correspondence to Timothy P.L. Roberts, PhD, Children's Hospital of Philadelphia, 34th and Civic Center Blvd, Neuroradiology, Suite 2115, Wood Building, Philadelphia, PA 19104.

- Indicates open access to non-subscribers at www.ajnr.org

http://dx.doi.org/10.3174/ajnr.A3754 evoked mismatch fields. ${ }^{6}$ Thus, part of the motivation of our present study is to identify neural substrates that support language and that are shared in SLI and ASD+LI.

Other evidence, however, suggests that the 2 disorders are distinct. For example, children with SLI make different types of errors than children with ASD+LI in nonword repetition tasks. ${ }^{7}$ Studies examining the superior longitudinal fasciculus, a major white matter pathway connecting the Broca and Wernicke areas and subserving language function, also suggest that SLI and ASD + LI are distinct. For

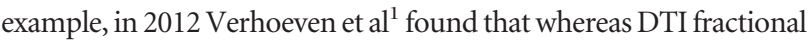
anisotropy (FA) measures of the superior longitudinal fasciculi were reduced in participants with SLI compared with age-matched control participants, FA differences were not observed between participants with ASD+LI and age-matched control participants (differences in $\mathrm{ADC}$, a measure equivalent to mean diffusivity [MD], were also not found between any of the groups). This SLI and ASD+LI white matter difference might be interpreted as suggesting a neurobiologic abnormality unique to SLI.

Recently, our group demonstrated elevated MD of the left hemi- 
sphere superior longitudinal fasciculus in ASD vs typically developing control participants, with group differences exacerbated by the degree of LI in ASD participants. ${ }^{8}$ To further investigate the above issues, in our present study, diffusion parameters of the superior longitudinal fasciculus in SLI were compared with typically developing and both ASD groups examined in Nagae et al. ${ }^{8}$ Given that in the study by Nagae et al ${ }^{8}$ group differences were most pronounced in the arcuate fasciculus, an area that corresponds with the temporal aspect of the superior longitudinal fasciculus, analyses focused on DTI parameters in this superior longitudinal fasciculus subregion.

It has been hypothesized that similarities in left hemisphere MD elevation in ASD+LI and SLI would be revealed in a main effect of LI. However, given an elevation in MD, even without LI, as reported by Nagae et al, ${ }^{8}$ the possibility of a main effect of ASD per se was also considered. Although the MD results in the typically developing and ASD groups have been reported by Nagae et $\mathrm{al},{ }^{8}$ in our study, this previous work was extended by incorporating the SLI cohort. Furthermore, and importantly, to probe a mechanistic underpinning of MD observations, and to identify potential differences in the cause of MD elevation, closer examination of the axial diffusivities (ADs) and radial diffusivities (RDs) was attempted as functions of LI, and of a diagnosis of ASD.

\section{MATERIALS AND METHODS}

Some DTI analyses of the typically developing and ASD groups in our study have been reported previously. ${ }^{8}$ In addition, magnetoencephalography measures of the M100 auditory response and the magnetic mismatch field have been previously reported for all participants. $^{6,9,10}$ All studies were performed with permission from our institutional review board, with the written informed consent of a parent and assent from participants.

\section{Participants}

Fourteen children with SLI (mean age, $9.73 \pm 2.69$ years; 8 boys) were recruited from the Center for Childhood Communication at the Children's Hospital of Philadelphia. Thirty-four participants with ASD were recruited through the Regional Autism Center at the Children's Hospital of Philadelphia: 16 with concomitant LI (mean age, $9.80 \pm 2.57$ years; 14 boys), and 18 without LI (mean age, $11.47 \pm 3.25 ; 16$ boys). A cohort of 25 age-matched control participants was also included (mean age, $11.42 \pm 2.92$ years, 16 boys). Groups did not differ in age $(P>.05)$.

Speech-language pathologists and neuropsychologists completed diagnostic and language assessments. Details of the inclusion and exclusion criteria for each group are reported in previous studies. ${ }^{6,8-10}$ Language was assessed by use of tests including the Clinical Evaluation of Language Fundamentals - Fourth Edition (CELF-4) ${ }^{11}$ and the Comprehensive Test of Phonological Processing. ${ }^{12}$ All referred ASD participants had a prior diagnosis of ASD, which was confirmed by the Autism Diagnostic Observation Schedule, ${ }^{13}$ the Krug Asperger's Disorder Index, ${ }^{14}$ the Social Responsiveness Scale, ${ }^{15}$ and parent report on the Social Communication Questionnaire. ${ }^{16}$ Global intelligence and academic achievement were assessed by use of the Wechsler Intelligence Scale for Children-Fourth Edition ${ }^{17}$ and the Wechsler Individual Achievement Test-Second Edition. ${ }^{18}$ All participants had a score of at least 75 on either the Perceptual Reasoning Index or Verbal Comprehension Index of the Wechsler Intelligence Scale for Children-Fourth Edition.

\section{Imaging}

DTI acquisition and postprocessing has been detailed elsewhere. ${ }^{8}$ In brief, acquisition parameters were as follows: isotropic 2-mmthick contiguous whole-brain acquisition performed on a $3 \mathrm{~T}$ magnet (Verio; Siemens, Erlangen, Germany); FOV, $25.6 \mathrm{~cm}$; matrix, $128 \times 128$; TR, 14,000 ms; TE, $70 \mathrm{~ms}$; a scheme with 80 contiguous sections and 30 diffusion-encoding gradient directions; $1 b=0\left(0 \mathrm{~s} / \mathrm{mm}^{2}\right), b \max =1000 \mathrm{~s} / \mathrm{mm}^{2}$; generalized autocalibrating partially parallel acquisition with an acceleration factor of 2.0. Postprocessing was performed by use of DTIStudio (Johns Hopkins University, Baltimore, Maryland). ${ }^{19}$ Streamline tractography of the right and left arcuate fasciculi was performed blind to the clinical group and following tractography protocol guidelines described by Wakana et $\mathrm{al}^{20}$ and Nagae et $\mathrm{al}^{8}$ by use of the fiber assignment by continuous tractography algorithm and a multiple region-of-interest inclusion criterion, with an FA threshold of 0.25 and a turning angle cutoff value of $70^{\circ}$. Figure 1 depicts left and right arcuate fasciculi tractograms from a representative 10-year-old boy from each group. MD was integrated over the entire tract length as described by Nagae et al, ${ }^{8}$ as were magnitudes of each eigenvalue, allowing computation of axial and $\mathrm{RDs}$. $\mathrm{AD}$, equivalent to eigenvalue $\lambda_{1}$, may be interpreted as diffusion along axon bundles. RD, defined as the mean of eigenvalues $\lambda_{2}$ and $\lambda_{3}$, may be interpreted as that component of diffusion in a plane perpendicular to the primary orientation of axonal bundles. $^{21,22}$

\section{Statistical Analysis}

Statistical analyses included a $2 \times 2$ ANOVA (general linear model) with factors of ASD and LI. Age and Perceptual Reasoning Index were included as covariates in all analyses. Given findings showing typically developing and ASD group differences in the left, but not right, hemisphere, ${ }^{8}$ analyses were conducted separately for the left and right arcuate fasciculus tracts. Finally, regression analyses examined associations between clinical measures-language and symptom ratings—and diffusivity measures.

\section{RESULTS}

As expected, children with ASD (both \pm LI) scored higher on the Social Responsiveness Scale than children in the typically developing and SLI groups $(P<.001)$. Social Responsiveness Scale scores did not differ between ASD - LI and ASD + LI $(P>.05)$ but were higher in those with SLI than typically developing subjects $(P<.01)$. CELF-4 Core Language Index scores were highest in the nonlanguage-impaired groups (ie, ASD-LI and typically developing, $P<.001)$. The SLI cohort performed better on the CELF-4 Core Language Index than the ASD + LI group $(P=.01)$. Typically developing and ASD-LI participants scored higher on the Full Scale Intelligence Quotient and Perceptual Reasoning Index than ASD +LI subjects $(P<.05)$.

In the left hemisphere, main effects of $\operatorname{ASD}(P=.03)$ and LI $(P=.04)$ were found on arcuate fasciculus MD (Fig 2). The interaction term was not significant $(P>.05)$. Significance of findings remained the same when age and Perceptual Reasoning Index were not included as covariates. No differences in left arcuate fasciculus FA were found.

To further understand the left hemisphere diffusion abnormalities, we examined $\mathrm{AD}$ and $\mathrm{RD}$. A main effect of ASD was 

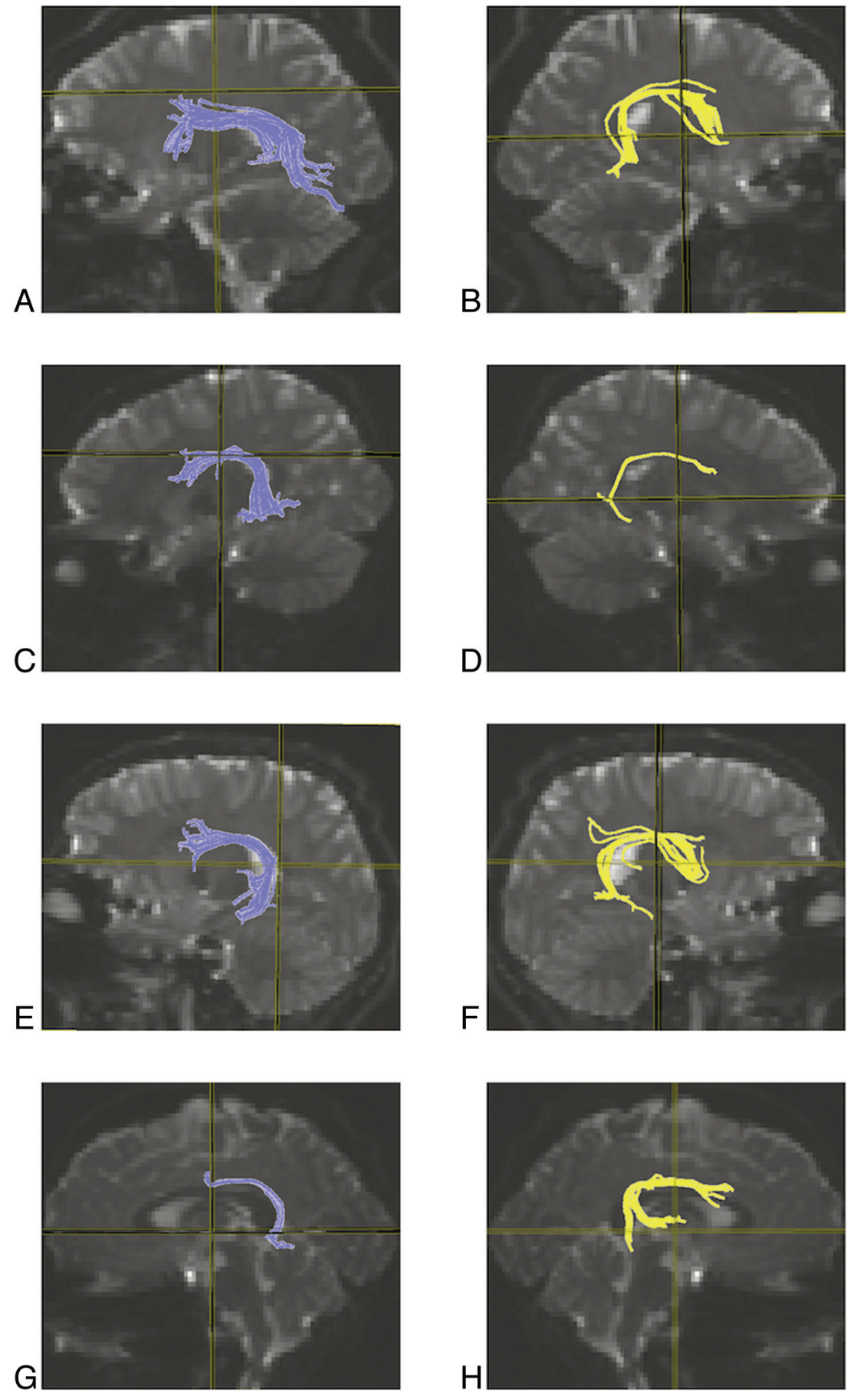

FIG 1. Streamline tractography of the arcuate fasciculus. Tractography of the left (left column; $A, C, E, G$ ) and right (right column; $B, D, F, H$ ) arcuate fasciculi in a 10-year-old male participant from each group (typically developing, $A-B ; A S D-L I, C-D ; A S D+L I, E-F ; S L I, G-H$ ).

found on $\mathrm{AD}(P<.001)$ but not $\mathrm{RD}(P=.61)$ (Fig 3). Conversely, there was a main effect of $\mathrm{LI}$ on $\mathrm{RD}(P=.02)$ but not $\mathrm{AD}(P=.55)$ (Fig 4). There was also a significant ASD by LI interaction on AD $(P=.05)$, revealing the highest $\mathrm{AD}$ in the $\mathrm{ASD}+\mathrm{LI}$ group. The interaction term was not significant for $\mathrm{RD}(P=.68)$.

No group differences were observed in the right hemisphere arcuate fasciculus for any of the $4 \mathrm{DTI}$ measures: $\mathrm{MD}, \mathrm{FA}, \mathrm{RD}$, or AD. The Table shows age- and Perceptual Reasoning Index-corrected marginal means for $\mathrm{MD}, \mathrm{FA}, \mathrm{AD}$, and $\mathrm{RD}$ for each hemisphere.
Hierarchic regression examined associations between clinical symptoms and diffusivity measures. Given the significant effects of age and Perceptual Reasoning Index on diffusivity, hierarchic regressions were performed with age entered first, Perceptual Reasoning Index second, and CELF-4 Core Language Index scores third, with left hemisphere arcuate fasciculus diffusivity parameters as the dependent measures (Table). CELF-4 Core Language Index scores predicted unique variance in left arcuate fasciculus $\mathrm{MD}[F(1,68)=7.83, P<.01]$, RD $[F(1,68)=5.00, P=.03]$, and $\mathrm{AD}$ $[F(1,68)=3.87, P=.05]$. Rerunning the above regressions for each subgroup, however, did not show relationships with CELF-4 Core Language Index for any of the individual groups. CELF-4 Core Language Index scores did not predict variance in FA $[F(1,68)=1.09, P=.30]$. Pooling the 2 language impaired subgroups (SLI and ASD $+\mathrm{LI}$ ), we observed a nonsignificant trend for an association between elevated $\mathrm{MD}$ and impaired language performance reflected in the CELF-4 Core Language In$\operatorname{dex}[F(1,25)=3.29, P=.08]$.

Regressions were also performed with age and Perceptual Reasoning Index entered into the first 2 blocks and Social Responsiveness Scale scores third. Social Responsiveness Scale scores predicted unique variance in $\operatorname{MD}[F(1,67)=3.92, P=.05]$ and $\mathrm{AD}[F(1,67)=6.70, P=.01]$. Social Responsiveness Scale scores did not predict the variance in $\operatorname{RD}[F(1,67)=0.68, P=.41]$ or FA $[F(1,67)=0.25, p=0.62]$. Similar to the CELF-4 Core Language Index analyses, regressions with Social Responsiveness Scale were not significant when groups were individually analyzed.

\section{DISCUSSION}

There were 2 main findings. First, main effects of both ASD and also LI were found on MD of the left hemisphere arcuate fasciculus, with both ASD and LI associated with elevated MD. At first, this finding appears to reflect a shared ASD and LI biologic substrate. However, a second finding showed a main effect of ASD on the axial, but not radial, component of diffusivity, and a main effect of LI on the radial, but not axial, component of diffusivity. As both $\mathrm{AD}$ and $\mathrm{RD}$ elevations could contribute to the observed MD elevations, this trend suggests that though both ASD and LI are associated with microstructural abnormalities in the left hemisphere arcuate fasciculus, the nature of these microstructural abnormalities is somewhat distinct. 


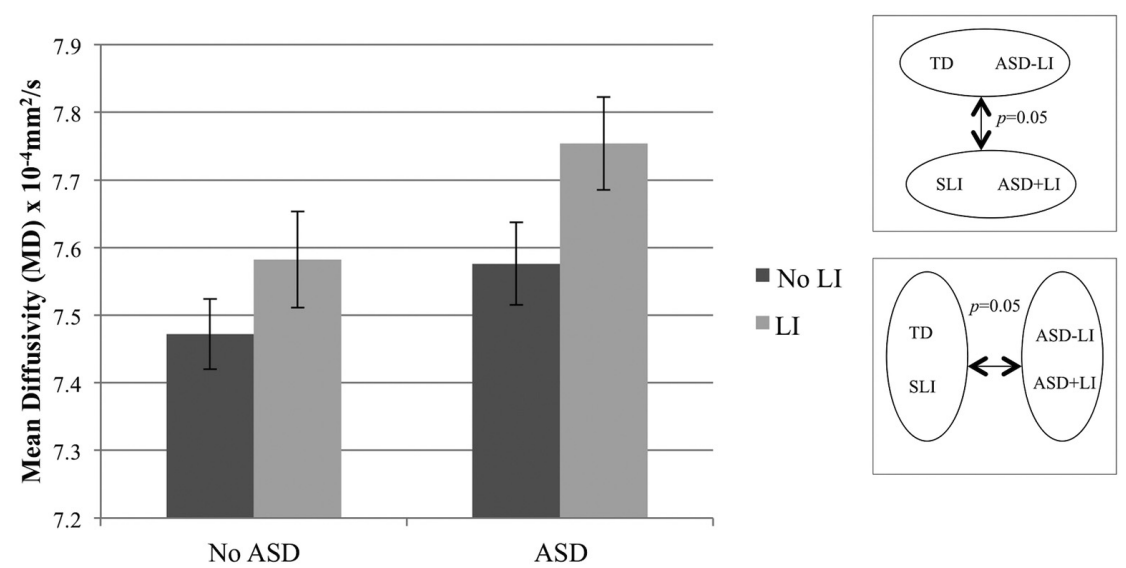

FIG 2. MD of the left arcuate fasciculus. ANOVA with factors of ASD $( \pm)$ and LI ( \pm ), and covarying age and Perceptual Reasoning Index, revealed significant main effects of ASD $(P=.03)$ and $\mathrm{LI}(P=.04)$.

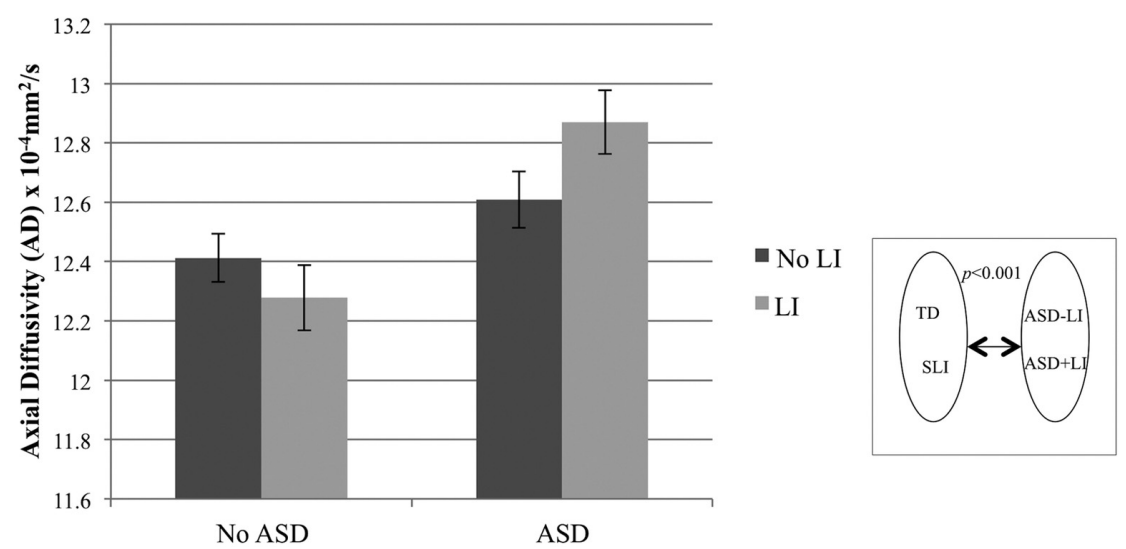

FIG 3. AD of the left arcuate fasciculus. ANOVA with factors of ASD ( \pm ) and LI ( \pm ), and covarying age and Perceptual Reasoning Index, revealed a main effect of ASD $(P<.001)$. There was also a significant ASD by $\mathrm{LI}$ interaction $(P=.05)$, revealing the greatest elevation in the ASD + LI group.

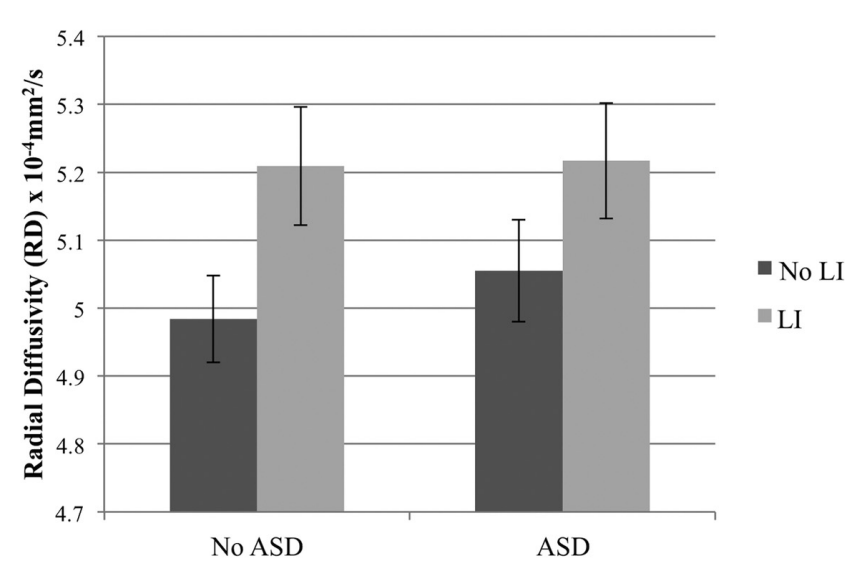

FIG 4. RD of the left arcuate fasciculus. ANOVA with factors of ASD ( \pm ) and LI ( \pm ), and covarying age and Perceptual Reasoning Index, revealed a significant main effect of $\mathrm{LI}(P=.02)$ on RD in the left arcuate fasciculus.

Diffusion imaging studies in ASD generally report elevated diffusivity and decreased FA in multiple brain areas, though the nature of arcuate fasciculus abnormalities is still in debate, as reviewed by Travers et al. ${ }^{21}$ Even less is known about the white matter integrity of children with SLI. Verhoeven et $\mathrm{al}^{1}$ compared DTI parameters of the superior longitudinal fasciculus in children with SLI and children with ASD+LI with healthy control participants. Contrary to present findings and previously reported ASD studies, ${ }^{8,21}$ the only significant finding in Verhoeven et $\mathrm{al}^{1}$ was decreased FA in SLI. Discrepancies between this study and our present study may be related to the approach by Verhoeven et al, ${ }^{1}$ namely to evaluate the entire superior longitudinal fasciculi tract rather than its subregions. Nonetheless, decreased FA is commonly associated with elevated MD; thus, apparent differences among studies may reflect differing sensitivities of these commonly used eigenvalue constructs rather than different underlying biologic processes. Furthermore, FA reflects a composite combination of eigenvalues perhaps obscuring opposing individual eigenvalue phenomena.

An additional finding emerges from attempting to understand the nature of the LI and ASD white matter abnormalities, with $\mathrm{AD}$ and $\mathrm{RD}$ analyzed separately (Figs 3-5). In our current study, $\mathrm{AD}$ was associated with ASD and RD with LI. That is, in both SLI and ASD + LI, elevated RD was observed, reinforcing the notion of similar anatomic substrates underlying LI in both populations. A main effect of ASD (and not $\mathrm{LI}$ ) on $\mathrm{AD}$, as well as the significant ASD by LI interaction in AD, however, suggests an additional contribution to a microstructural anomaly in ASD+LI (above and beyond that in SLI). Thus, in short, although ASD+LI and SLI may share a microstructural anomaly of elevated $\mathrm{RD}$, they differ in AD. As Fig 5 illustrates, this mechanism leads to a "doubly" elevated MD in the ASD+LI cohort.

Consistent with the general linear model main effects, linguistic abilities (reflected in the CELF-4 Core Language Index) correlated with left hemisphere arcuate fasciculus RD when assessed across all participants. In addition, an association with $\mathrm{AD}$ was also resolved. However, this relationship between language skills and $\mathrm{AD}$ (not predicted by the general linear model analyses) was most likely driven by the fact that more than half of the LI participants also have ASD and, thus, elevated AD. Indeed, the relationship between CELF-4 Core Language Index scores and AD did not 


\begin{tabular}{|c|c|c|c|c|c|c|c|c|}
\hline & \multicolumn{2}{|c|}{ TD } & \multicolumn{2}{|c|}{ SLI } & \multicolumn{2}{|c|}{ ASD-LI } & \multicolumn{2}{|c|}{ ASD+LI } \\
\hline & LH & RH & LH & RH & LH & RH & LH & RH \\
\hline $\begin{array}{c}\mathrm{MD} \times 10^{-4} \\
\mathrm{~mm}^{2} / \mathrm{s}\end{array}$ & $7.472 \pm 0.052^{a}$ & $7.606 \pm 0.059^{a}$ & $7.582 \pm 0.071$ & $7.602 \pm 0.078$ & $7.576 \pm 0.061^{a}$ & $7.664 \pm 0.070^{a}$ & $7.754 \pm 0.069^{a}$ & $7.749 \pm 0.079^{a}$ \\
\hline FA & $.542 \pm 0.005^{\mathrm{a}}$ & $.528 \pm 0.007^{\mathrm{a}}$ & $0.525 \pm 0.007$ & $.519 \pm 0.009$ & $.543 \pm 0.006^{a}$ & $.524 \pm 0.008^{\mathrm{a}}$ & $.535 \pm 0.007^{a}$ & $.504 \pm 0.009^{a}$ \\
\hline $\mathrm{RD}$ & $4.984 \pm 0.064$ & $5.140 \pm 0.076$ & $5.209 \pm 0.087$ & $5.233 \pm 0.100$ & $5.055 \pm 0.075$ & $5.255 \pm 0.089$ & $5.217 \pm 0.085$ & $5.404 \pm 0.100$ \\
\hline$A D$ & $12.412 \pm 0.081$ & $12.513 \pm 0.085$ & $12.278 \pm 0.110$ & $12.310 \pm 0.112$ & $12.609 \pm 0.095$ & $12.589 \pm 0.100$ & $12.870 \pm 0.107$ & $12.461 \pm 0.113$ \\
\hline
\end{tabular}

Note:-All are marginal mean measures \pm SEM projected to a mean age of 10.81 years and a Perceptual Reasoning Index (PRI) score of 101.92 in the left hemisphere; mean age was 10.75 years, and PRI score was 101.97 in the right hemisphere.

TD indicates typically developing, LH, left hemisphere; $\mathrm{RH}$, right hemisphere.

${ }^{\text {a }}$ Partially reported in Nagae et al. ${ }^{8}$

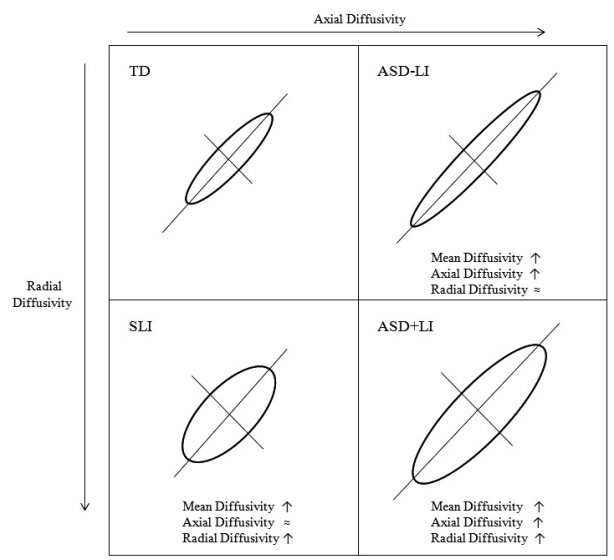

FIG 5. A schematic of the diffusion changes accompanying ASD and LI. Mean diffusivity was elevated in LI and ASD. Children with ASD had elevated AD (right boxes) and, thus, elevated MD. Patients with LI (bottom boxes) had elevated MD because of increased RD. Patients with ASD and LI had the highest MD values because of combined effects of both elevated RD and AD.

persist when ASD symptom severity was included in the model. Conversely, the relationship between linguistic skills and RD strengthened when measures of ASD were included in the model. However, none of the associations remained significant when individual subpopulations were considered, though there was a nonsignificant trend $(P=.08)$ in the association between MD and CELF-4 Core Language Index when evaluated over all children with LI (SLI and ASD+LI groups pooled). As an analogy, Social Responsiveness Scale scores correlated positively with AD and were unrelated to RD when evaluated across all participants, but again, associations were not significant for individual subpopulations, perhaps reflecting the need for yet larger sample sizes in each group.

We encountered limitations comparing our current results with the previous literature. A review of DTI studies in ASD found that only approximately one-third of studies reported AD and RD findings. ${ }^{21}$ Fletcher et $\mathrm{al}^{23}$ reported elevated MD and RD in the left arcuate fasciculus of 10 adolescents with ASD, and no differences in FA or AD. However, the wide range of participants' linguistic skills (composite CELF-3 scores ranged from 50-111 and were significantly lower than those of an age-matched control group) makes it difficult to distinguish the separate effects of ASD and LI on diffusivity. In a similar fashion, the limited data available on SLI offer limited insight into the effects of LI on white matter microstructural integrity. Verhoeven et $\mathrm{al}^{1}$ did report a positive correlation between the Word Classes Receptive subtest of the CELF-4 and mean FA in the superior longitudinal fasciculus of adolescents with SLI. However, no such correlations were found in the ASD+LI group, and the authors did not report AD or RD.

The biologic interpretation of these microstructural anomalies remains speculative. It is tempting to consider elevated RD as reflecting immaturely bundled axons (with greater interaxonal space for $\mathrm{RD}$ ). Increases in $\mathrm{AD}$ are perhaps less intuitive. However, in white matter with fiber crossings, alterations to the pattern of these crossing fibers or, indeed, the RD of component fibers can both manifest as increased $\mathrm{AD} .^{24}$ In mouse models, decreased $\mathrm{AD}$ and increased $\mathrm{RD}$ have been associated with acute axon and myelin damage, respectively. ${ }^{25-27}$ However, these findings may not directly translate to humans, particularly in cases of chronic white matter microstructural damage. Pathologic increases of AD have also been reported. In a small study of adults with acute optic neuritis, Naismith et $\mathrm{al}^{28}$ reported decreased $\mathrm{AD}$ within 1 month of onset, but there was substantially increased $\mathrm{AD}$ relative to control participants 1 year later. Indeed, widespread increased AD has been demonstrated in Alzheimer disease, ${ }^{29}$ and in the right frontal tracts of patients with Parkinson disease. ${ }^{30}$ Moreover, $\mathrm{RD}$ and $\mathrm{AD}$ seem to be far less specific in areas of chronic microstructural damage. In a study of diffusion in the spinal cord of patients with multiple sclerosis, Klawiter et al ${ }^{31}$ reported associations between $\mathrm{RD}$ and both levels of demyelination and axonal damage, but no relationship between axonal damage and AD. Although DTI is sensitive to alterations to white matter microstructure, the technique alone cannot provide a specific mechanism for the observed changes.

Finally, although our present findings are intriguing, a limitation of our study was that small sample sizes prevented the inclusion of handedness and sex as factors or inclusion/exclusion criteria. Furthermore, although the sample size evaluated in our study is a non-meager 73, it is clear that fine-grained microstructural characterization of both ASD and LI substrates, common and distinct, will require much larger samples, perhaps a need served by emerging multi-institutional data sharing initiatives such as the National Database for Autism Research (http://ndar. nih.gov/).

\section{CONCLUSIONS}

Elevations in the MD of the left hemisphere arcuate fasciculus were associated with both ASD and LI, potentially suggesting a similar biologic substrate. Closer inspection of the microstruc- 
tural anomalies, however, suggested that elevated AD was associated with ASD and elevated RD with LI. In comparing ASD+LI with SLI, it is clear that both are similar in that they share elevated $\mathrm{RD}$, but that they are distinct in that only ASD $+\mathrm{LI}$ additionally manifests elevated $\mathrm{AD}$, and thus $\mathrm{ASD}+\mathrm{LI}$ shows the most profound elevations in MD.

\section{ACKNOWLEDGMENTS}

We acknowledge Rachel Golembski, Jamie Rundio, and Peter Lam for technical assistance. Dr. Roberts thanks the Oberkircher family for the Oberkircher Family Endowed Chair in Pediatric Radiology. The authors would like to thank Thorsten Feiweier of Siemens Medical Solutions for the diffusion imaging pulse sequence.

Disclosures: Timothy Roberts—RELATED: National Institutes of Health (NIH), ${ }^{*}$ Nancy Lurie Marks Family Foundation (NLMFF), ${ }^{*}$ Comments: R01DC008871, P30HD026979, a grant from NLMFF; UNRELATED: Consultancy: Elekta, Prism Clinical Imaging. Deborah Zarnow—RELATED: Grant: NIH R01DC008871, ${ }^{*}$ Nancy Lurie Marks Family Foundation, ${ }^{*} \mathrm{NIH}$ P3OHD026979.* Lidia Nagae—RELATED: Grant: NIH, ${ }^{*}$ NLMFF, ${ }^{*}$ Comments: NIH R01DC008871, ${ }^{\star}$ NLMFF, ${ }^{\star}$ NIH P30HD026979* (Dr. Roberts's grants); UNRELATED: Grants/Grants Pending: The Radiological Society of North America Fellow Grant Award. * Lisa Blaskey—RELATED: Grant: NIH, ${ }^{*}$ Nancy Lurie Marks Family Foundation, ${ }^{*}$ Comments: NIH grant R01DC008871 (TR), NLMFF. Cynthia SolotRELATED: Grant: to Tim Roberts. * Susan Levy—RELATED: Grant: NIH, ${ }^{*}$ Comments: Grants: R01DC008871, P30HD026979; Other: Nancy Lurie Marks Family Foundation, ${ }^{*}$ Comments: Private foundation. Jeffrey Berman-UNRELATED: Consultancy: McGowan Associates, Comments: MR compatibility testing unrelated to manuscript. J. Christopher Edgar-RELATED: Grant: NIH, ${ }^{\star}$ Comments: Grant to Dr. Roberts to study auditory processes in autism. *Money paid to institution.

\section{REFERENCES}

1. Verhoeven JS, Rommel N, Prodi E, et al. Is there a common neuroanatomical substrate of language deficit between autism spectrum disorder and specific language impairment? Cereb Cortex 2012;22: 2263-71

2. Bishop DV. Overlaps between autism and language impairment: phenomimicry or shared etiology? Behav Genet 2010;40:618-29

3. Tager-Flusberg H, Joseph RM. Identifying neurocognitive phenotypes in autism. Philos Trans R Soc Lond B Biol Sci 2003;358:303-14

4. Conti-Ramsden G, Simkin Z, Botting N. The prevalence of autistic spectrum disorders in adolescents with a history of specific language impairment (SLI). J Child Psychol Psychiatry 2006;47:621-28

5. De Fossé L, Hodge SM, Makris N, et al. Language-association cortex asymmetry in autism and specific language impairment. Ann $\mathrm{Neu}$ rol 2004;56:757-66

6. Roberts TP, Heiken K, Kahn SY, et al. Delayed magnetic mismatch negativity field, but not auditory M100 response, in specific language impairment. Neuroreport 2012;23:463-68

7. Williams D, Payne H, Marshall C. Non-word repetition impairment in autism and specific language impairment: evidence for distinct underlying cognitive causes. J Autism Dev Disord 2013;43:404-17

8. Nagae LM, Zarnow DM, Blaskey L, et al. Elevated mean diffusivity in the left hemisphere superior longitudinal fasciculus in autism spectrum disorders increases with more profound language impairment. AJNR Am J Neuroradiol 2012;33:1720-25

9. Roberts TP, Khan SY, Rey M, et al. MEG detection of delayed auditory evoked responses in autism spectrum disorders: towards an imaging biomarker for autism. Autism Res 2010;3:8-18
10. Roberts TP, Cannon KM, Tavabi K, et al. Auditory magnetic mismatch field latency: a biomarker for language impairment in autism. Biol Psychiatry 2011;70:263-69

11. Semel EM, Wiig, EH, Secord, W. Clinical Evaluation of Language Fundamentals (CELF-4). San Antonio, Texas: The Psychological Corporation; 2003

12. Wagner RK, Torgesen JK, Rashotte CA, et al. Comprehensive Test of Phonological Processing. Austin, Texas: Pro-Ed; 1999

13. Lord $\mathrm{C}$, Risi S, Lambrecht $\mathrm{L}$, et al. The autism diagnostic observation schedule-generic: a standard measure of social and communication deficits associated with the spectrum of autism. J Autism Dev Disord 2000;30:205-23

14. Krug D, Arick, JR. Krug Asperger's Disorder Index. Los Angeles: Western Psychological Services; 2003

15. Constantino J, Gruber, CP. Social Responsiveness Scale (SRS). Los Angeles: Western Psychological Services; 2005

16. Rutter M, Bailey, A., Lloyd, C. SCQ: Social Communication Questionnaire. Los Angeles: Western Psychological Services; 2003

17. Wechsler D. Wechsler Intelligence Scale for Children. San Antonio, Texas: The Psychological Corporation; 2003

18. Wechsler D. Wechsler Individual Achievement Test. San Antonio, Texas: The Psychological Corporation; 2001

19. Jiang H, van Zijl PC, Kim J, et al. DTIStudio: resource program for diffusion tensor computation and fiber bundle tracking. Comput Methods Programs Biomed 2006;81:106-16

20. Wakana S, Caprihan A, Panzenboeck MM, et al. Reproducibility of quantitative tractography methods applied to cerebral white matter. Neuroimage 2007;36:630-44

21. Travers BG, Adluru N, Ennis C, et al. Diffusion tensor imaging in autism spectrum disorder: a review. Autism Res 2012;5:289-313

22. Wozniak JR, Lim KO. Advances in white matter imaging: a review of in vivo magnetic resonance methodologies and their applicability to the study of development and aging. Neurosci Biobehav Rev 2006;30:762-74

23. Fletcher PT, Whitaker RT, Tao R, et al. Microstructural connectivity of the arcuate fasciculus in adolescents with high-functioning autism. Neuroimage 2010;51:1117-25

24. Wheeler-Kingshott CA, Cercignani M. About "axial” and "radial" diffusivities. Magn Reson Med 2009;61:1255-60

25. Song SK, Yoshino J, Le TQ, et al. Demyelination increases radial diffusivity in corpus callosum of mouse brain. Neuroimage 2005;26:132-40

26. Song SK, Sun SW, Ju WK, et al. Diffusion tensor imaging detects and differentiates axon and myelin degeneration in mouse optic nerve after retinal ischemia. Neuroimage 2003;20:1714-22

27. Song SK, Sun SW, Ramsbottom MJ, et al. Dysmyelination revealed through MRI as increased radial (but unchanged axial) diffusion of water. Neuroimage 2002;17:1429-36

28. Naismith RT, Xu J, Tutlam NT, et al. Disability in optic neuritis correlates with diffusion tensor-derived directional diffusivities. Neurology 2009;72:589-94

29. Acosta-Cabronero J, Alley S, Williams GB, et al. Diffusion tensor metrics as biomarkers in Alzheimer's disease. PLoS One 2012;7:e49072

30. Theilmann RJ, Reed JD, Song DD, et al. White-matter changes correlate with cognitive functioning in Parkinson's disease. Front $\mathrm{Neu}$ rol 2013;4:37

31. Klawiter EC, Schmidt RE, Trinkaus K, et al. Radial diffusivity predicts demyelination in ex vivo multiple sclerosis spinal cords. $\mathrm{Neu}$ roimage 2011;55:1454-60 